\title{
ALPINE: zanubrutinib versus ibrutinib in relapsed/refractory chronic lymphocytic leukemia/small lymphocytic lymphoma
}

\author{
Peter Hillmen*,1, Jennifer R Brown², Barbara F Eichhorst ${ }^{3}$, Nicole Lamanna ${ }^{4}$, Susan M \\ $\mathrm{O}^{\prime}$ Brien $^{5}$, Lugui Qiu ${ }^{6}$, Tommi Salmi ${ }^{7}$, James Hilger ${ }^{7}$, Kenneth $\mathrm{Wu}^{7}$, Aileen Cohen ${ }^{7}$, Jane \\ Huang $^{7}$ \& Constantine S Tam ${ }^{8}$ \\ ${ }^{1}$ Department of Haematology, Leeds Teaching Hospitals NHS Trust, Level 3 Bexley Wing, St James's University Hospital, Leeds LS9 \\ 7TF, UK \\ ${ }^{2}$ Dana-Farber Cancer Institute, Boston, MA 02215, USA \\ ${ }^{3}$ Department of Internal Medicine, University of Cologne, Cologne 50923, Germany \\ ${ }^{4}$ Herbert Irving Comprehensive Cancer Center, Columbia University, New York, NY 10032, USA \\ ${ }^{5}$ Chao Family Comprehensive Cancer Center, University of California, Orange, CA 92868, USA \\ ${ }^{6}$ Institute of Hematology \& Blood Diseases Hospital, Chinese Academy of Medical Sciences \& Peking Union Medical College, \\ Tianjin 300020, PR China \\ ${ }^{7}$ BeiGene USA, Inc., San Mateo, CA 94403, USA \\ ${ }^{8}$ Peter MacCallum Cancer Centre, St Vincent's Hospital, University of Melbourne, Melbourne, Victoria 3000, Australia and \\ University of Melbourne, Parkville, Victoria 3052, Australia \\ *Author for correspondence: Tel.: +1 0113343 8604; peter.hillmen@nhs.net
}

Treatment standards for chronic lymphocytic leukemia (CLL) have been transformed with the advent of effective inhibitors of B-cell receptor signaling such as ibrutinib - a first-in-class inhibitor of BTK. Offtarget kinase inhibitions by ibrutinib are thought to contribute to its adverse events. Zanubrutinib is a next-generation BTK inhibitor with minimal off-target effects, sustained BTK occupancy in peripheral blood mononuclear cells and lymph nodes from patients with B-cell malignancies and promising responses in patients with CLL. Described here is a head-to-head Phase III study comparing the efficacy and safety of zanubrutinib with those of ibrutinib in patients with CLL/small lymphocytic lymphoma in the relapsed/refractory setting.

First draft submitted: 20 December 2019; Accepted for publication: 17 January 2020; Published online: 24 March 2020

Keywords: Bruton tyrosine kinase $\bullet$ BTK inhibitor $\bullet$ chronic lymphocytic leukemia • clinical trials $\bullet$ comparative effectiveness • hematologic/leukemia • ibrutinib • zanubrutinib

Here, we describe an ongoing, head-to-head Phase III study comparing the efficacy of zanubrutinib (BGB3111 ) with that of ibrutinib in patients with relapsed or refractory $(R / R)$ chronic lymphocytic leukemia/small lymphocytic lymphoma (CLL/SLL) as measured by overall response rate (ORR), progression-free survival (PFS), time to treatment failure and overall survival (OS). This study also includes an exploratory analysis of clinical outcomes according to prognostic and predictive biomarkers (ClinicalTrials.gov identifier NCT03734016).

\section{Background \& rationale}

CLL is an indolent yet fatal malignancy of B lymphocytes, with morbidity and mortality related to immunosuppressive and myelosuppressive sequelae of progressive disease [1,2]. Treatment standards for CLL/SLL have transformed since the advent of effective inhibitors of B-cell receptor signaling, allowing for nonchemotherapy-based treatment regimens for patients with previously treated CLL/SLL [2]. Blockade of the B-cell receptor signaling cascade by inhibition of either BTK or PI3K $\delta$ causes profound inhibition of proliferative signaling from CLL/SLL cell-host interactions and results in frequent and durable responses in patients with R/R CLL/SLL [3]. However, the use of $\mathrm{PI} 3 \mathrm{~K} \delta$ inhibitors is often limited by toxicities, including hepatotoxicity, colitis and infectious complications, particularly when used in combination with other agents [4]. Venetoclax, an orally bioavailable inhibitor of BCL-2, was

Future 8 Medicine 
approved in 2016 by the United States Food and Drug Administration for the treatment of patients with CLL/SLL and a $17 \mathrm{p}$ deletion (del[17p]) who had received at least one prior therapy, based on a study in 107 patients with $\mathrm{R} / \mathrm{R}$ del[17p] CLL/SLL with an ORR of 79\% [5,6]. Recently, the US label for venetoclax was expanded to include the treatment of all adult patients with CLL/SLL, with or without $17 \mathrm{p}$ deletion, who have received at least one prior therapy. This change was based on results from the MURANO trial in R/R CLL/SLL that demonstrated a benefit in 2-year PFS of $84.9 \%$ in the patients who received venetoclax plus rituximab versus a 2-year PFS of $36.3 \%$ in patients who received bendamustine plus rituximab [6,7].

Ibrutinib (Imbruvica), the first-in-class BTK inhibitor, was approved for use in CLL in 2014 and has a favorable tolerability profile when compared with conventional therapies. In the initial Phase Ib/II study of patients with R/R CLL ( $\mathrm{n}=85)$, in which patients received ibrutinib at a daily dose of $420 \mathrm{mg}(\mathrm{n}=51)$ or $840 \mathrm{mg}(\mathrm{n}=34)$, the ORR, defined as achieving a partial response (PR) or better, was identical at $71 \%$ for patients at either dose [8]. An additional 20 and $15 \%$ of patients in the two dosing groups, respectively, had a PR with lymphocytosis (PR-L). The responses observed were independent of clinical and genomic risk factors including del[17p] status. At 26 months, the estimated PFS rate was $75 \%$, and the OS rate was $83 \%[8]$.

In the Phase III RESONATE study that compared ibrutinib with ofatumumab (an anti-CD20 monoclonal antibody) in 391 patients with R/R CLL, ibrutinib was found to significantly improve PFS (ibrutinib: median PFS not reached; ofatumumab: median PFS of 8.1 months; $\mathrm{p}<0.001)$ at a median follow-up of 9.4 months and OS: $90 \%$ with ibrutinib versus $81 \%$ with ofatumumab at 12 months $(\mathrm{p}=0.005)$ [9]. Overall response rate (PR and complete response [CR]) for ibrutinib were 70 and $43 \%$ by investigator and independent review, respectively. An additional $15 \%$ for investigator and $20 \%$ by independent review patients had a PR-L making the ORR including PR-L $83 \%$ by investigator and $63 \%$ by independent review [9]. The most common adverse events (AEs) (that occurred in $>20 \%$ of patients) seen in patients treated with ibrutinib included diarrhea (48\%), fatigue (28\%), nausea $(26 \%)$, pyrexia $(24 \%)$, anemia $(23 \%)$ and neutropenia $(22 \%)$. Of note, $14 \%$ of patients experienced grade 3 or higher neutropenia [9,10]. Lastly, some cases of ibrutinib-induced tumor lysis syndrome have been reported [11].

Long term follow-up of the RESONATE study demonstrated significantly longer PFS and OS for ibrutinib than ofatumumab (HR: 0.148; 95\% CI: 0.113-0.196; $p$ < 0.0001) with a median follow-up of 65.3 months (range: 0.3-71.6 months) with the estimated PFS for the ibrutinib arm of 40 versus $3 \%$ in the ofatumumab arm at the 60 month landmark. Responses deepened over time with an ORR of $91 \%$ for ibrutinib and $9 \%$ of patients with a $\mathrm{CR}$ or $\mathrm{CR}$ with incomplete bone marrow recovery [9,12].

At the final analysis of RESONATE, the most commonly reported AEs were in line with previous reports. Prevalence of most AEs decreased over time with the exception of hypertension and bruising. Overall 10\% of patients treated on the ibrutinib arm experienced major hemorrhage, $21 \%$ experienced hypertension and $12 \%$ experienced atrial fibrillation of any grade. Most $(92 \%)$ patients who experienced atrial fibrillation had risk factors including cardiovascular disease or hypertension [12].

\section{Zanubrutinib}

Zanubrutinib (BGB-3111) is a potent, specific and irreversible BTK inhibitor with a favorable pharmacological and pharmacokinetic (PK) profile. Zanubrutinib has greater selectivity in relative inhibition of BTK versus off-target tyrosine kinases seen with ibrutinib [13,14], including EGFR, FGR, FRK, HER2, HER4, ITK, JAK 3, LCK and TEC, which are thought to cause diarrhea, thrombocytopenia, bleeding, atrial fibrillation, rash and fatigue [14,15].

Full occupancy of BTK in peripheral blood mononuclear cells was achieved in all patients in the first-in-human Phase I BGB-3111-AU-003 study (ClinicalTrials.gov identifier NCT02343120), while occupancy in lymph node tissue was assessed at $160 \mathrm{mg}$ twice daily and $320 \mathrm{mg}$ once daily [14]. At the 160-mg twice-daily dose, full BTK occupancy was observed at trough, suggesting that sustained target occupancy could be achieved in lymph nodes and peripheral blood resulting in the inhibition of BTK on a continuous basis. The $100 \%$ BTK inhibition observed with zanubrutinib in both blood and lymph nodes is hypothesized to maximize the chances for deep and sustained remissions in CLL and other hematologic malignancies. There is now extensive experience at the 160-mg twice-daily and 320-mg once-daily doses; both schedules show a high level of activity without compromising the tolerability profile. The oral 160-mg twice-daily dose has been selected as the recommended dose for registration studies, based on sustained target occupancy, high rates of objective response in multiple types of B-cell malignancies and a favorable safety and tolerability profile.

In the Phase I BGB-3111-AU-003 study, 78 patients with CLL/SLL treated with zanubrutinib monotherapy were evaluable for efficacy [14]. After a median follow-up of 13.7 months (range: 0.4-30.5 months), the ORR 
by investigator was $96.2 \%$ (75/78) (95\% CI: 89.2-99.2), including two patients (2.6\%) with a CR, 63 (80.8\%) with a PR and 10 (12.8\%) with a PR-L. All 22 treatment-naive CLL/SLL patients responded with one CR, 18 PR and 3 patients with PR-L. In addition, all efficacy-evaluable patients with either del[17p] or TP53 mutation responded [14]. In keeping with the drug's high selectivity for BTK inhibition, zanubrutinib demonstrated a favorable toxicity profile with few off-target effects such as diarrhea and rash (observed in only $\sim 20 \%$ of patients and all grade 1 or 2 events) [14].

A safety analysis of pooled data from six zanubrutinib monotherapy trials showed that zanubrutinib has been generally well tolerated in patients across various B-cell malignancies [16]. A total of 682 patients with non-Hodgkin lymphoma, CLL/SLL, Waldenström macroglobulinemia, hairy cell leukemia and Richter's transformation were included (data cutoff range: September 2018-February 2019). The median age was 64 years (range: 20-90) and $68 \%$ were male. The median duration of zanubrutinib exposure was 13 months (25th-75th percentile: 620 months). The most common AEs of grade 3 or higher were decreased neutrophil count (14\%), anemia (8\%), neutropenia (7\%), pneumonia (5\%), decreased platelet count (4\%), lung infection (4\%) and hypertension (3\%). The cumulative safety profile of zanubrutinib indicates that the events of interest for this class of BTK inhibitors were infrequent: atrial fibrillation/flutter (1.9\%), grade $\geq 3$ hemorrhage $(2.1 \%)$ and grade $\geq 3$ diarrhea $(0.9 \%)$. Among the 13 patients with atrial fibrillation/flutter, most had risk factors including cardiovascular disease $(\mathrm{n}=7)$, hypertension $(n=4)$, hyperlipidemia $(n=4)$, concurrent infection $(n=4)$ and prior atrial fibrillation $(n=1)$. Myalgias or arthralgias occurred in $<10 \%$ of patients [16].

Zanubrutinib is a potent and selective BTK inhibitor with optimized pharmacologic properties relative to what has been reported for ibrutinib, including ready oral bioavailability and enhanced selectivity for BTK [14]. The Phase III study BGB-3111-305 was designed to compare the efficacy and safety of zanubrutinib with the first-in-class BTK inhibitor ibrutinib in a broadly defined population of patients with R/R CLL/SLL. The intent is to compare these agents not only in terms of achievement of disease control, as measured by ORR, but also to compare these two BTK inhibitors as sustained treatment for CLL/SLL in regard to PFS, DoR and, importantly, safety and tolerability measures of the agents as prolonged therapy.

\section{Study design}

Trial

This Phase III, randomized, open-label study compares zanubrutinib versus ibrutinib treatment in approximately 600 patients with R/R CLL/SLL. Refractory disease is defined as disease progression or no objective response within 6 months of the last treatment; relapsed disease is defined as progression or relapse $>6$ months after the last treatment. The primary objective of the study is to compare the efficacy of zanubrutinib versus ibrutinib defined by ORR as determined by investigator.

\section{Key eligibility criteria}

Eligible patients must be aged $\geq 18$ years, be able to provide written informed consent and have a confirmed diagnosis of CLL or SLL that meets the International Workshop on Chronic Lymphocytic Leukemia criteria for disease and for requiring treatment [17]. Patients must have R/R disease with one or more prior systemic therapies consisting of at least two cycles of treatment, measurable disease, an Eastern Cooperative Oncology Group performance status of $0-2$, adequate bone marrow function with or without growth factors and transfusions, adequate organ function and a life expectancy $\geq 6$ months.

Patients will be excluded for any one of the following reasons: known prolymphocytic leukemia or Richter's transformation, clinically significant cardiovascular disease, QTcF prolongation $>480 \mathrm{~ms}$, history of a severe bleeding disorder, stroke or intracranial hemorrhage within 180 days, other malignancy within the past 3 years (except skin cancer, superficial bladder cancer or carcinoma in situ of the cervix or breast), debilitating pulmonary disease, recent major surgery, ongoing infections or any prior treatment with a BTK inhibitor.

\section{Study procedures}

Patients will be randomized in a 1:1 manner to receive zanubrutinib $160 \mathrm{mg}$ orally twice daily in Arm A or ibrutinib $420 \mathrm{mg}$ orally once daily in Arm B (Figure 1). Randomization will be stratified by age ( $<65 \mathrm{vs} \geq 65$ years), geographic region (China vs other participating countries), refractory status (yes or no) and centrally tested $\operatorname{del}[17 \mathrm{p}] /$ TP53 mutation status (present or absent). Patients will remain on the study drug until disease progression, unacceptable toxicity, treatment consent withdrawal or study termination. 


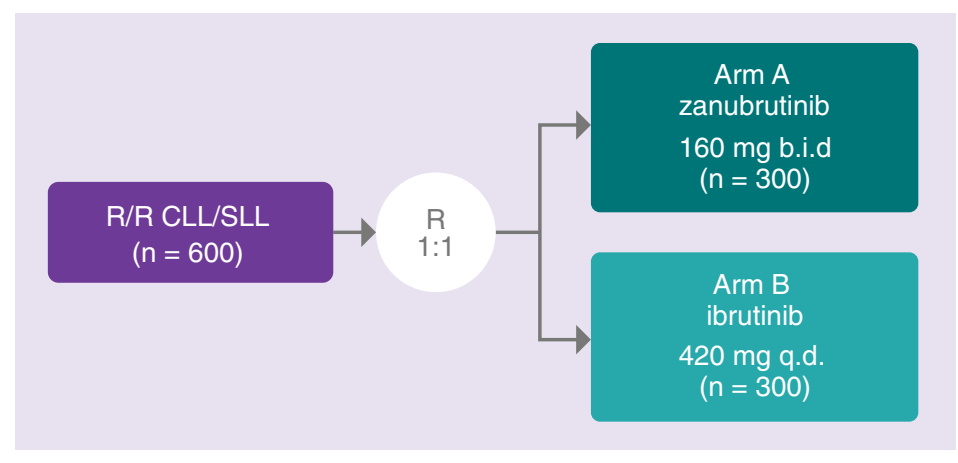

Figure 1. ALPINE study design. bid: Twice daily; CLL/SLL: Chronic lymphocytic leukemia/small lymphocytic lymphoma; EOT: End of treatment; PD: Progressive disease; qd: Once daily; R: Randomize; R/R: Relapsed/refractory.

In the event of grade $\geq 3$ nonhematologic toxicities, grade $\geq 3$ neutropenia with infection or fever, grade $\geq 3$ thrombocytopenia associated with bleeding or grade 4 thrombocytopenia or neutropenia lasting 10 consecutive days or more considered to be related to zanubrutinib, drug will be withheld until resolution of any related toxicities. Dosing can be resumed following an interruption of up to 28 days but at a reduced dose following the second and third AEs ( $80 \mathrm{mg}$ twice daily and once daily, respectively). Zanubrutinib is to be discontinued in patients who experience their fourth drug-related AE. The local ibrutinib label should be followed for dose modification. In general, ibrutinib will be interrupted for grade $\geq 3$ nonhematologic toxicities, grade $\geq 3$ neutropenia with infection or fever or grade 4 hematologic toxicities. Once the toxicity has resolved to grade 1 or baseline, ibrutinib therapy may be reinitiated at the starting dose. For the second and third recurrences of the toxicity, the dose will be reduced to 280 and $140 \mathrm{mg}$ once daily, respectively.

\section{Outcome measures}

The primary objective is to compare the efficacy of zanubrutinib with that of ibrutinib as measured by the ORR. Secondary objectives include PFS, duration of response (DoR), time to treatment failure (TTF), rate of PR-L or higher, OS, safety parameters, and patient-reported outcomes. Patients with a potential CR will undergo a bone marrow examination to confirm CR or complete response with incomplete bone marrow recovery (CRi) and possibly to determine the presence or absence of minimal residual disease.

Disease response will be assessed per the 2008 International Workshop on Chronic Lymphocytic Leukemia guidelines [17], with modification for treatment-related lymphocytosis [18] for patients with CLL and per Lugano classification for non-Hodgkin lymphoma [19] for patients with SLL. Rates of PR-L or better will be assessed as a secondary efficacy end point; treatment with BTK inhibitors may lead to lymphocytosis due to redistribution of leukemia cells from the lymphoid compartment to blood, which is not a sign of treatment failure or disease progression and has no bearing on treatment outcome [20].

Assessments of safety will include AEs, serious adverse events, clinical laboratory tests, physical examinations, electrocardiograms and vital signs. AEs will be graded for severity per the current version (4.03) of the National Cancer Institute Common Terminology Criteria for Adverse Events. Hematologic adverse events will be graded according to the grading scale for hematologic toxicity in CLL [17]. An independent data monitoring committee will periodically monitor safety data and perform the interim efficacy analysis.

Patient-reported outcomes assessed on the study include the European quality of life 5-D, 5-level health questionnaire (EQ-5D-5L) and the European Organisation for Research and Treatment of Cancer quality of life cancer core questionnaire (EORTC QLQ-C30). In select countries, there will be an optional device-based evaluation of quality of life and activity.

\section{Planned study period}

Treatment with zanubrutinib and ibrutinib will be open label. The first patient was dosed on 1 November 2018. Based on enrollment prediction, the study duration is estimated to be 51 months.

\section{Statistics}

\section{Analysis sets}

The primary analysis set for all efficacy analyses is the Intent-to-Treat Analysis Set (all randomized patients). Noninferiority (NI) testing of ORR will also be performed using the Per-Protocol Analysis Set, which will include 
patients who receive any dose of study drug and do not have any major protocol deviations. The Safety Analysis Set will include all patients who receive any dose of study drug. The PK Analysis Set will include all zanubrutinib-treated patients who have at least one post-dose drug concentration.

\section{Planned sample size \& methods of analysis}

Approximately 600 patients will be enrolled and randomized in a 1:1 ratio to receive either zanubrutinib or ibrutinib. The primary efficacy analysis of ORR (PR or better, defined as CR/CRi + PR + nodular PR) by investigator review will be tested first for NI of zanubrutinib to ibrutinib. Superiority will be subsequently tested if $\mathrm{NI}$ is demonstrated.

There will be one interim analysis approximately 12 months after $69 \%$ of patients have been randomized. The final analysis will occur approximately 12 months after 600 patients have been randomized, which is expected to occur 3 years after the first patient has been randomized. A Cochran-Mantel-Haenszel test for the response ratio, adjusted for the four randomization stratification factors, will be performed to test the hypothesis. CochranMantel-Haenszel response ratio with its 95\% Wald CI will be estimated as magnitude of treatment effect. The Clopper-Pearson 95\% CIs will be calculated for ORR for each treatment group.

PFS by investigator review will be also tested for NI using stratified log-rank test if the primary objective is met and the hazard ratio will be also estimated using stratified Cox regression. Distribution of PFS, OS, and DoR will be estimated by Kaplan-Meier method for each arm. Cox regression will be used to estimate the hazard ratios (HRs) for OS by INV.

\section{Conclusion}

Targeting the BTK signaling pathway has proven to be highly efficacious for patients with CLL/SLL. Ibrutinib, the first-in-class BTK inhibitor, is a highly effective agent. However, ibrutinib is associated with AEs, including atrial fibrillation, diarrhea, arthralgias [15], many of which are thought to be the result of off-target kinase inhibitions [13]. Zanubrutinib, a next-generation BTK inhibitor, aims to improve upon both the safety and efficacy of ibrutinib. Selected for its high specificity for BTK, favorable pharmacology and PK, zanubrutinib has the potential to further improve outcomes in patients with CLL/SLL. This head-to-head Phase III trial is designed to compare the safety and efficacy of zanubrutinib with ibrutinib in patients with CLL/SLL.

\section{Executive summary}

\section{BTK inhibition in chronic lymphocytic leukemia}

- B-cell receptor signaling, crucial for the survival and proliferation of chronic lymphocytic leukemia (CLL) cells, is reliant on several different tyrosine kinases, including BTK.

- Blockade of the B-cell receptor signaling cascade by the first-in-class BTK inhibitor therapy ibrutinib has resulted in frequent and durable responses in patients with CLL.

- Ibrutinib is a once-daily oral therapy indicated for treatment of adult patients with CLL/small lymphocytic lymphoma (SLL), including patients with CLL with del[17p]/TP53 mutation status.

- Ibrutinib has a highly favorable tolerability profile when compared with conventional therapies, but is associated with adverse events such as hemorrhage, infections, cytopenia, cardiac arrythmia, hypertension, second primary malignancies, tumor lysis syndrome, diarrhea and rash, some of which have been attributed to off-target kinase activity of this drug.

Zanubrutinib

- Zanubrutinib is an irreversible BTK inhibitor with a favorable pharmacologic and pharmacokinetic profile.

- Zanubrutinib has achieved complete, $100 \%$ BTK inhibition in both blood and lymph nodes, which is believed to maximize the chances for deep and sustained remissions in CLL and other hematologic malignancies.

- Zanubrutinib has greater selectivity in relative inhibition of BTK versus off-target tyrosine kinases seen with ibrutinib, including EGFR, FGR, FRK, HER2, HER4, ITK, JAK 3, LCK and TEC, which are thought to cause diarrhea, thrombocytopenia, bleeding, atrial fibrillation, rash and fatigue.

Phase III trial

- This is a head-to-head Phase III, randomized, open-label, multicenter study comparing the efficacy and safety of zanubrutinib with those of ibrutinib in patients with CLL/SLL in the relapsed/refractory setting.

- Approximately 600 patients will be randomized in a 1:1 manner to receive zanubrutinib 160 mg orally twice daily or ibrutinib $420 \mathrm{mg}$ orally once daily.

Objectives

- The primary objective of this study is to compare the efficacy of zanubrutinib with that of ibrutinib, as measured by overall response rate in patients with relapsed/refractory CLL/SLL. 
- Secondary objectives are to further compare the efficacy of zanubrutinib with that of ibrutinib, as measured by progression-free survival, duration of response, time to treatment failure, rate of partial response with lymphocytosis or higher, overall survival and patient-reported outcomes.

- Secondary objectives also include safety comparisons between the two study drugs.

Statistics

- The primary end point of overall response rate will be compared between the two study drugs using a Cochran-Mantel-Haenszel test of the response ratio of the two treatment groups after adjustment for stratification factors.

- The secondary efficacy end points of progression-free survival will be analyzed using a stratified log-rank test; comparisons of other secondary efficacy end points between treatment arms will be descriptive, and no hypothesis testing will be performed.

Conclusion

- Zanubrutinib is a second-generation BTK inhibitor designed for higher efficacy and improved specificity; therefore, it has the potential to further improve outcomes in patients with CLL/SLL.

- Results of this study will spotlight the potential benefits and implications on safety and efficacy of zanubrutinib, a kinase inhibitor that was designed to have selectivity for BTK with fewer off-target effects.

Author contributions

P Hillmen, JR Brown, BF Eichhorst, N Lamanna, SM O'Brien, L Qiu, J Hilger, and CS Tam were responsible for study design. P Hillmen, JR Brown, N Lamanna, SM O'Brien, L Qiu, and CS Tam have contributed or will contribute to patient enrollment. BeiGene was involved in study design, compilation of data, and statistical analysis. All authors had full access to all of the data, contributed to data interpretation and analysis, carefully reviewed the manuscript and approved the final version. The corresponding author, $\mathrm{P}$ Hillmen, had final responsibility to submit for publication.

Financial \& competing interests disclosure

P Hillmen is a consultant/advisor for Acerta, Janssen and AbbVie; received research funding from AbbVie, Pharmacyclics, Janssen, Gilead and Roche; and received honoraria from AbbVie and Janssen. JR Brown is a consultant/advisor for AbbVie, Acerta, AstraZeneca, BeiGene, Catapult Therapeutics, Dynamo Therapeutics, Genentech/Roche, Gilead, Juno/Celgene, Kite, Loxo, Novartis, Pfizer, Pharmacyclics, Sunesis, TG Therapeutics and Verastem; received research funding from Gilead, Loxo, Sun and Verastem; received honoraria from Janssen and Teva; and is a member of the data safety monitoring board for Morphosys and Invectys. BF Eichhorst is a consultant/advisor for Janssen, Roche, Novartis, AbbVie, Gilead, Celgene and ArQule; received research funding from Janssen, Roche, AbbVie, Gilead and BeiGene; received speakers' bureau honoraria from Janssen, Roche, Novartis, AbbVie, Gilead and Celgene; and is a member of the advisory board for Janssen, Roche, Novartis, AbbVie, Gilead and Celgene. N Lamanna is a consultant/advisor for AbbVie, AstraZeneca, BeiGene, Acerta, Gilead, Genentech, Juno, Janssen, Octapharma, Pharmacyclics and Celgene; received research funding from AbbVie, AstraZeneca, BeiGene, Juno, Infinity/Verastem, Ming, TG Therapeutics and Oncternal; and is a member of the advisory board for AstraZeneca, BeiGene, AbbVie, Gilead, Celgene, Genentech, Juno and Pharmacyclics. SM O’Brien is a consultant/advisor for Amgen, Astellas, Celgene, GlaxoSmithKline, Janssen Oncology, Aptose Biosciences, Vaniam Group LLC, AbbVie, Alexion, Verastem, Eisai, Gilead, Pharmacyclics, TG Therapeutics, Pfizer and Sunesis; and received research funding from Kite, Regeneron, Acerta, Gilead, Pharmacyclics, TG Therapeutics, Pfizer and Sunesis. L Qiu has nothing to disclose. T Salmi, J Hilger, K Wu, A Cohen and J Huang are employed by and have equity ownership with BeiGene. CS Tam received research funding from Janssen and AbbVie and honoraria from Janssen, Abbvie, BeiGene, Novartis and Roche. The authors have no other relevant affiliations or financial involvement with any organization or entity with a financial interest in or financial conflict with the subject matter or materials discussed in the manuscript apart from those disclosed.

This clinical trial is supported by BeiGene Company Ltd, San Mateo, CA, USA. Medical writing and editorial assistance was funded by BeiGene and was provided, under the direction of the authors, by A Richardson, Bio Connections, LLC and A Daisy Goodrich.

Ethical conduct of research

The authors state that they have obtained appropriate institutional review board approval or have followed the principles outlined in the Declaration of Helsinki for all human or animal experimental investigations. In addition, for investigations involving human subjects, informed consent has been obtained from the participants involved.

Open access

This work is licensed under the Attribution-NonCommercial-NoDerivatives 4.0 Unported License. To view a copy of this license, visit http://creativecommons.org/licenses/by-nc-nd/4.0/ 


\section{References}

Papers of special note have been highlighted as: $\bullet$ of interest; $\bullet \bullet$ of considerable interest

1. Riches JC, Ramsay AG, Gribben JG. Immune reconstitution in chronic lymphocytic leukemia. Curr. Hematol. Malig. Rep. 7(1), 13-20 (2012).

2. Kipps TJ, Stevenson FK, Wu CJ et al. Chronic lymphocytic leukaemia. Nat. Rev. Dis. Primers 3, 16096 (2017).

3. Jerkeman M, Hallek M, Dreyling M et al. Targeting of B-cell receptor signalling in B-cell malignancies. J. Intern. Med. 282(5), 415-428 (2017).

4. Zydelig (idelasib), package insert. Gilead Sciences, CA, USA (2018).

5. Stilgenbauer S, Eichhorst B, Schetelig J et al. Venetoclax in relapsed or refractory chronic lymphocytic leukaemia with $17 \mathrm{p}$ deletion: a multicentre, open-label, phase 2 study. Lancet Oncol. 17(6), 768-778 (2016).

6. Venclexta (venetoclax tablets), package insert. AbbVie Inc., IL, USA (2019).

7. Seymour JF, Kipps TJ, Eichhorst B et al. Venetoclax-rituximab in relapsed or refractory chronic lymphocytic leukemia. N. Engl. J. Med. 378(12), 1107-1120 (2018).

8. Byrd JC, Furman RR, Coutre SE et al. Targeting BTK with ibrutinib in relapsed chronic lymphocytic leukemia. N. Engl. J. Med. 369(1), 32-42 (2013).

-• Established ibrutinib as a therapy for chronic lymphocytic leukemia/small lymphocytic lymphoma.

9. Byrd JC, Brown JR, O’Brien S et al. Ibrutinib versus ofatumumab in previously treated chronic lymphoid leukemia. N. Engl. J. Med. 371(3), 213-223 (2014).

10. Kaur V, Swami A. Ibrutinib in CLL: a focus on adverse events, resistance, and novel approaches beyond ibrutinib. Ann. Hematol. 96(7), 1175-1184 (2017).

11. Titus-Rains KS, Brown JN, Hammond JM. Ibrutinib-associated tumor lysis syndrome in chronic lymphocytic leukemia/small lymphocytic lymphoma and mantle cell lymphoma: A case series and review of the literature. J. Oncol. Pharm. Pract. 24(7), 544-559 (2018) (Epub ahead of print).

- $\quad$ Provides evidence of ibrutinib-induced tumor lysis syndrome.

12. Munir T, Brown JR, O'Brien S et al. Final analysis from RESONATE: up to six years of follow-up on ibrutinib in patients with previously treated chronic lymphocytic leukemia or small lymphocytic lymphoma.. Am. J. Hematol. 94(12), 1353-1363 (2019) (Epub ahead of print).

13. Honigberg LA, Smith AM, Sirisawad M et al. The Bruton tyrosine kinase inhibitor PCI-32765 blocks B-cell activation and is efficacious in models of autoimmune disease and B-cell malignancy. Proc. Natl Acad. Sci. USA 107(29), 13075-13080 (2010).

- Introduces the Bruton tyrosine kinase inhibitor ibrutinib.

14. Tam CS, Trotman J, Opat S et al. Phase 1 study of selective BTK inhibitor zanubrutinib in B-cell malignancies and safety and efficacy evaluation in CLL. Blood 134(11), 851-859 (2019).

-• Introduces second generation Bruton tyrosine kinase inhibitor zanubrutinib in chronic lymphocytic leukemia/small lymphocytic lymphoma.

15. Coutre SE, Byrd JC, Hillmen P et al. Long-term safety of single-agent ibrutinib in patients with chronic lymphocytic leukemia in 3 pivotal studies. Blood Adv. 3(12), 1799-1807 (2019).

-• Provides long-term safety data on ibrutinib.

16. Tam C, Opat S, Zhu J et al. Pooled analysis of safety data from monotherapy studies of the Bruton tyrosine kinase (BTK) inhibitor, zanubrutinib (BGB-3111), in B-cell malignancies: PS1159. Hemasphere European Hematology Association Annual Meeting, Amsterdam, 3, 526 (2019) (PosterPS1159).

- Pooled safety data across zanubrutinib monotherapy studies demonstrating general tolerability across hematologic malignancies.

17. Hallek M, Cheson BD, Carovsky D et al. Guidelines for the diagnosis and treatment of chronic lymphocytic leukemia: a report from the International Workshop on Chronic Lymphocytic Leukemia updating the National Cancer Institute-Working Group 1996 guidelines. Blood 111(12), 5446-5456 (2008).

18. Cheson BD, Byrd JC, Rai KR et al. Novel targeted agents and the need to refine clinical end points in chronic lymphocytic leukemia. J. Clin. Oncol. 30(23), 2820-2822 (2012).

19. Cheson BD, Fisher RI, Barrington SF et al. Recommendations for initial evaluation, staging, and response assessment of Hodgkin and non-Hodgkin lymphoma: the Lugano classification. J. Clin. Oncol. 32(27), 3059-3068 (2014).

20. Woyach JA, Smucker K, Smith LL et al. Prolonged lymphocytosis during ibrutinib therapy is associated with distinct molecular characteristics and does not indicate a suboptimal response to therapy. Blood 123(12), 1810-1817 (2014). 
